\title{
Modulation of Electronic States near Electrodes in Graphene Transistors Observed by Operando Photoelectron Nanospectroscopy
}

\author{
Hirokazu Fukidome, ${ }^{1 *}$ Kazutoshi Funakubo, ${ }^{1}$ Naoka Nagamura, ${ }^{2,3}$ Koji Horiba, ${ }^{4}$ \\ Yasunori Tateno, ${ }^{5}$ Masaharu Oshima, ${ }^{6}$ and Maki Suemitsu ${ }^{1}$ \\ ${ }^{1}$ Research Institute of Electrical Communication, Tohoku University, \\ 2-1-1 Katahira, Aoba-ku, Sendai, Miyagi 980-8577, Japan \\ ${ }^{2}$ Research Center for Advanced Measurement and Characterization, National Institute for Materials Science, \\ 1-2-1 Sengen, Tsukuba, Ibaraki 305-0047, Japan \\ ${ }^{3}$ PRESTO, Japan Science and Technology Agency, \\ 4-1-8 Honcho, Kawaguchi, Saitama 332-0012, Japan \\ ${ }^{4}$ Photon Factory, Institute of Materials Structure Science, High Energy Accelerator Research Organization, \\ 1-1 Oho, Tsukuba, Ibaraki 305-0801, Japan \\ ${ }^{5}$ Sumitomo Electric Industries, \\ 1, Taya-cho, Sakae-ku, Yokohama, Kanagawa 244-8508, Japan \\ ${ }^{6}$ Synchrotron Radiation Research Organization, Institute for Solid State Physics, The University of Tokyo, \\ 7-3-1 Hongo, Bunkyo-ku, Tokyo 113-8656, Japan
}

(Received February 12, 2019; accepted May 14, 2019)

Keywords: graphene, aluminum, natural oxidation, transistor, gate dielectrics, electrodes

The 2D crystal of $\mathrm{sp}^{2}$-bonded carbon atoms (graphene) is suitable for high-speed device applications owing to its excellent electronic properties and ultrathinness that suppresses the socalled short-channel effects. Unfortunately, however, graphene transistors have not exhibited high device performance as anticipated from their excellent electronic properties. One of the main causes is a high susceptibility of electronic properties to surrounding interfaces. Here, we show operando, i.e., bias-controlled, X-ray photoelectron nanospectroscopy on a transistor using epitaxial graphene on $\mathrm{SiC}$ as a channel. The operando photoelectron nanospectroscopy revealed that two types of unfavorable doping occur at the interfaces with electrodes, which can deteriorate the device performance of graphene transistors. Metals of source and drain electrodes are in direct contact with graphene. An equilibration between graphene and the metal produces a wide charge transfer region owing to a limited density of states near the Dirac point of graphene. The gate electric field permeates just outside the gate edge. The permeated electric field induces carrier doping into graphene just outside the gate edge. Thus, the operando photoelectron nanospectroscopy is proven useful in the science-based development of advanced devices.

*Corresponding author: e-mail: fukidome@riec.tohoku.ac.jp https://doi.org/10.18494/SAM.2019.2327 


\section{Introduction}

The ultrathin 2D crystal, graphene, possesses excellent electronic properties, such as the highest carrier mobility and saturation velocity of carriers in solids. ${ }^{(1-3)}$ Besides, the so-called short-channel effects are expected to be suppressed because of the ultrathinness of graphene. These promise high device performance of graphene-based devices. Unfortunately, however, graphene-based devices, such as graphene field-effect transistors (GFETs), do not exhibit high device performance. $^{(3)}$ One of the causes of the degraded device performance is parasitic resistances, such as contact resistances between graphene and source/drain and resistances in access regions, which are ungated regions of graphene between the gate and the source or drain electrode. ${ }^{(4)}$ These parasitic resistances become marked in GFETs, ironically because the channel resistance of graphene is much lower than those of conventional semiconductors, e.g., $\mathrm{Si}$, owing to high carrier mobilities. Therefore, controlling the interfaces is vital for realizing high-performance graphene-based devices. For that purpose, it is necessary to understand the mechanism of how the interfaces modulate the electronic states of graphene.

The microscopic investigation of the electronic states of devices is effective in understanding the modulation of electronic states at the interfaces. We have developed X-ray spectromicroscopies, such as photoelectron nanospectroscopy for investigating valence bands ${ }^{(5,6)}$ and X-ray absorption spectromicroscopy for conduction bands, ${ }^{(7,8)}$ for the surfaces and interfaces of devices under bias applications, i.e., operando X-ray spectromicroscopies. These operando spectromicroscopies enabled us to nanoscopically probe electronic states at surfaces and interfaces in advanced devices, such as GFETs and transistors ${ }^{(5,7)}$ using $2 \mathrm{D}$ electron gas at an $\mathrm{AlGaN} / \mathrm{GaN}$ interface (GaN-HEMT). ${ }^{(6)}$ The results obtained by these operando spectromicroscopies contribute to revealing the operation mechanisms of advanced devices. For instance, we quantitatively revealed the details of surface electron trapping in GaN-HEMT, which causes the operation instabilities of GaN-HEMT. ${ }^{(6)}$

In this paper, we present the operando photoelectron nanospectroscopy study on the modulation of the electronic states of a graphene transistor near the electrodes due to the interface of graphene with the contact metal of the source or drain electrode and the permeation of the gate electric field.

\section{Materials and Methods}

Epitaxial graphene was formed on Si-terminated $\mathrm{SiC}(0001)$ by annealing in $\mathrm{Ar}$ ambient at $1873 \mathrm{~K}$ for $10 \mathrm{~min}$ as a channel for GFET. ${ }^{(9-11)}$ The epitaxial graphene thus formed exhibits high quality. This is because there is a negligible D band in a Raman spectrum. The G' band is narrow. The full width at half maximum (FWHM) is $39 \mathrm{~cm}^{-1}$. The FWHM indicates that epitaxial graphene is a high-quality monolayer. ${ }^{(9,10)}$

We have fabricated a transistor using epitaxial graphene on $\mathrm{SiC}(0001)$ as a channel [Fig. 1(b)]. Figure 1(c) shows the drain current $\left(I_{d}\right)$ and transconductance vs gate bias $\left(V_{g}\right)$ curves of a graphene transistor using epitaxial graphene on $\mathrm{SiC}$ as a channel (EG-GFET). The $I_{d}-V_{g}$ curves clearly demonstrated the ambipolarity that arises from the zero bandgap of graphene. ${ }^{(1)}$ The gate bias at the $I_{d}$ minimum, i.e., where the Fermi level $\left(E_{F}\right)$ coincides with the Dirac point, 


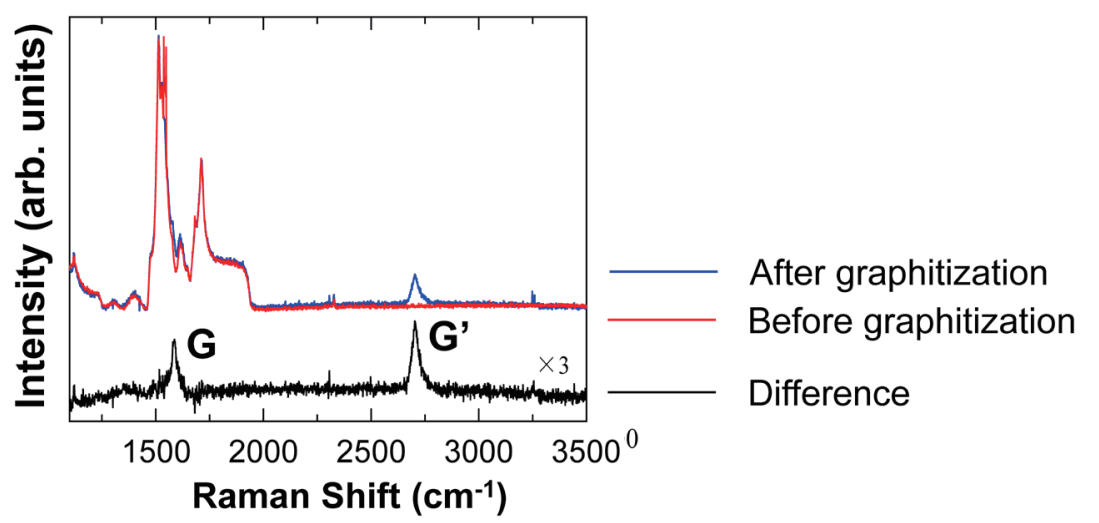

(a)

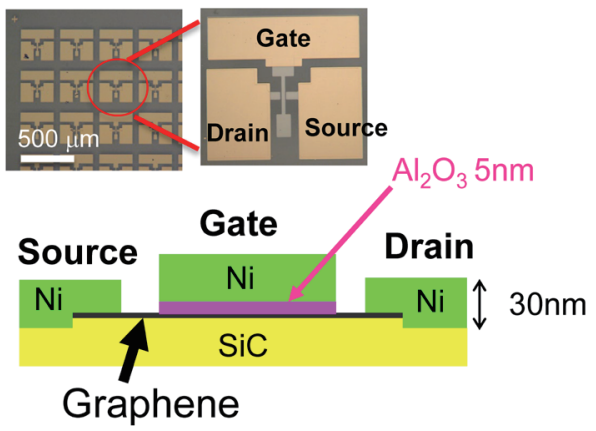

(b)

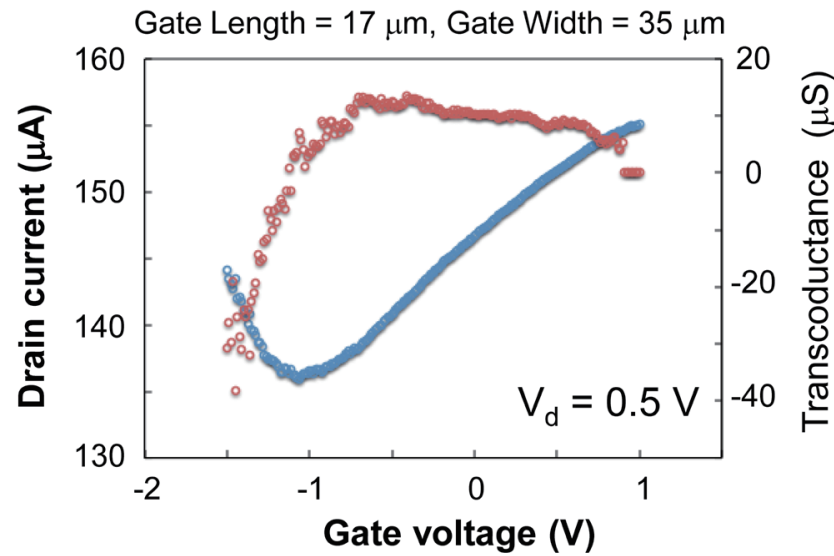

(c)

Fig. 1. (Color online) (a) Raman spectra of epitaxial graphene on the Si-terminated $\mathrm{SiC}(0001)$ before and after graphitization, and difference spectra. (b) Electrical characterization of EG-GFET. (c) $I_{d}-V_{g}$ curves of EG-FGET.

is around $-1 \mathrm{~V}$. This indicates that graphene is electron-doped. The electron doping is due to electron transfer from a buffer layer, which is situated between epitaxial graphene and $\mathrm{SiC} .{ }^{(9)}$ The estimated carrier mobility is $1360 \mathrm{~cm}^{2} / \mathrm{Vs}$.

Our operando photoelectron nanospectroscopy was done by using 3D nano-ESCA installed at BL07LSU at SPring- $8 .{ }^{(5,6,12-14)}$ The spatial resolution is $70 \mathrm{~nm}$, which is one of the highest resolutions of photoelectron spectroscopy in the world. The 3D nano-ESCA manifested its power in the mechanistic studies for the advanced electronic devices ${ }^{(5,6)}$ as well as complex surface structures of micro-electromechanical systems (MEMS). ${ }^{(13)}$

\section{Results and Discussion}

EG-GFET has two types of contact with electrodes. One is the contact of metal with epitaxial graphene for the source or drain electrode. The other is the contact of gate dielectrics with epitaxial graphene for the gate electrode. Here, we present the studies on the modulation of electronic states concerning the contact of metal and the permeated electric field of the gate electrode in separate subsections. 


\subsection{Metal contact}

The contact of epitaxial graphene on $\mathrm{SiC}$ with metals for the source or drain electrode is anticipated to bring about charge transfer at epitaxial graphene and the metals. This is because we demonstrated the interfacial charge transfer region between the exfoliated graphene and the metal for the source and drain in a transistor using the exfoliated graphene as a channel. ${ }^{(15)}$

In fact, our photoelectron nanospectroscopy revealed that the charge transfer region is formed also at the interface between the electrode metal and the epitaxial graphene on $\mathrm{SiC}$, as shown in Fig. 2. Figure 2(a) shows a typical spatially resolved C 1s spectrum of graphene, taken by our photoelectron nanospectroscopy. The spectrum consists of three peaks. ${ }^{(13,16)}$ The main peak at $\sim 284.7 \mathrm{eV}$ is due to graphene, whereas the peaks at $\sim 284.0$ and $\sim 285.7 \mathrm{eV}$ are ascribable to $\mathrm{SiC}$ and a buffer layer, which is a precursor of graphene and is partially bound to $\mathrm{SiC}^{(13,16)}$ The binding energy of the main peak, i.e., the energy difference between the Fermi energy $\left(E_{F}\right)$ and $\mathrm{C} 1 \mathrm{~s}$ core level of graphene, gives us information on the electronic state of epitaxial graphene on SiC. For instance, the carrier doping in graphene is inferred from the binding energy. The binding energy of electrically neutral graphite, which is regarded as the nondoped graphene, is $284.42 \mathrm{eV}^{(17)}$ The binding of energy of the epitaxial graphene is larger than that of the nondoped graphene. It is natural to conclude that the epitaxial graphene is electrondoped. The electron doping is consistent with our electrical characterization of EG-GFET [Fig. 1(c)], and also with a previous study involving angle-resolved photoelectron spectroscopy. The electron doping is ascribable to the presence of the buffer layer that causes the electron transfer to graphene. ${ }^{(18)}$ Near the metal contact, the binding energy of the graphene peak decreased over $1 \mu \mathrm{m}$, as shown in Fig. 2(b). The binding energy of the graphene peak is the energy difference between the $\mathrm{C} 1 \mathrm{~s}$ core level and $E_{F}$. The observed decrease near the metal contact means that

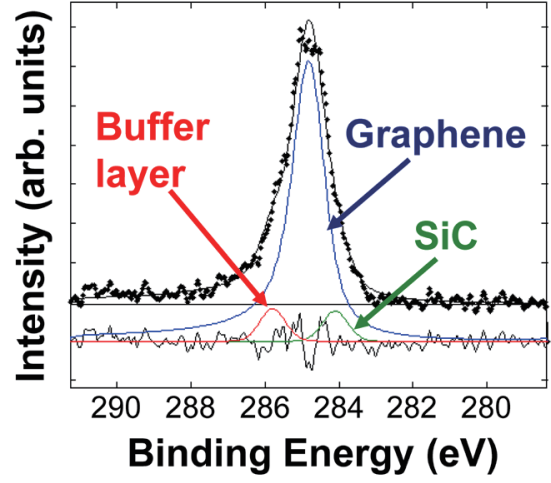

(a)

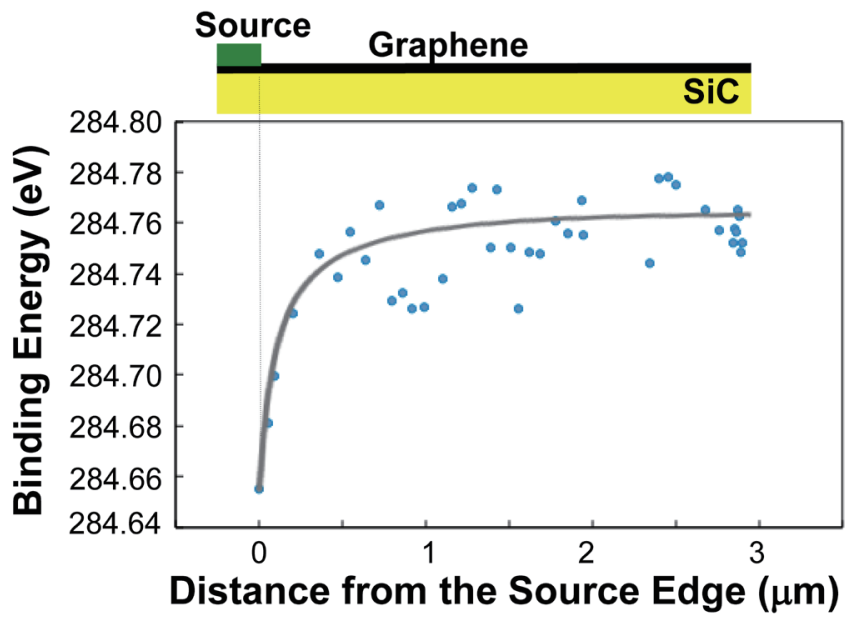

(b)

Fig. 2. (Color online) (a) Typical C 1s spectrum of epitaxial graphene on the Si-terminated SiC(0001) and (b) spatial change in the binding energy of the graphene peak near the source electrode. 
the $E_{F}$ of graphene decreased near the metal contact. Hole doping into graphene from the contact metal is plausible because the work function of $\mathrm{Ni}$ used as a contact metal $(5.2 \mathrm{eV})$ is larger than that of graphene $(4.5 \mathrm{eV})$. It is thus clarified that graphene is hole-doped by the presence of the contact metal in EG-FET, as in the case of the transistor using the exfoliated graphene as the channel. ${ }^{(15)}$

The charge transfer occurred owing to the equilibration between graphene and the contact metal. The limited density of states near the Dirac point, where the conduction band minimum touches the valence band top of graphene, makes the charge transfer region wider, ${ }^{(15,19)}$ whereas a charge transfer region to equilibrate is within $0.1 \mathrm{~nm}$ in the case of metal-metal contact because of a high density of states. The charge transfer region was semiquantitatively evaluated according to a previous theory within the framework of the Thomas-Fermi model using density functional calculations. ${ }^{(20)}$ According to the previous theory, the spatial distribution of potential graphene at the interface with the contact metal is expressed as

$$
V(x) \sim \frac{\Delta E}{\left(\sqrt{\frac{x}{l_{s}}+0.016}+0.787\right)^{1 / 2}\left(\frac{x}{l_{s}}+1.194\right)^{1 / 4}} .
$$

Here, $\Delta E$ is the boundary potential constant derived from the work functions calculated for the close-packed surface of the metal electrode and for graphene. $l_{S}=8.10 \times 10^{-2} \times \kappa / \Delta E$ indicates a scaling length, where $\kappa$ is an effective dielectric constant in a graphene device structure. ${ }^{(21)}$ Our experimental data were fitted by using Eq. (1), as shown in the solid line in Fig. 2(b). As a result, we obtained the following values: $\Delta E=0.29 \mathrm{eV}, \kappa=60$, and $l_{s}=16 \mathrm{~nm}$. The obtained $\Delta E$ is almost the same as that obtained in the case of the exfoliated graphene in contact with $\mathrm{Ni}^{(15)}$ Thus, the obtained $\Delta E$ is reasonable. On the other hand, the obtained $\kappa$ is larger than that $(\sim 16)$ estimated for the exfoliated graphene on $\mathrm{SiO}_{2}$ in contact with $\mathrm{Ni}$ in the same manner. There are two possible reasons for the difference in $\kappa$. One is a higher dielectric constant of $\mathrm{SiC}$ than that of $\mathrm{SiO}_{2}$. The other reason is the presence of polar materials, such as water molecules and hydroxyl groups, at the interfaces surrounding graphene. The charge transfer region can affect the device performance of EG-GFET because it can vary the access resistance of EG-GFET.

\subsection{Permeated gate field}

The gate bias application through the gate dielectrics is the heart of the transistor operation. We newly found by using our operando photoelectron nanospectroscopy that the permeation of the electric field formed during gate bias application changes the carrier concentration in graphene just outside the gate edge, as shown in Fig. 3. Figure 3(a) shows that the binding energy of graphene decreases just outside the gate edge. This indicates the decreased amount of electron doping near the gate edge. Besides, the observed decrease does not depend on the gate bias. 


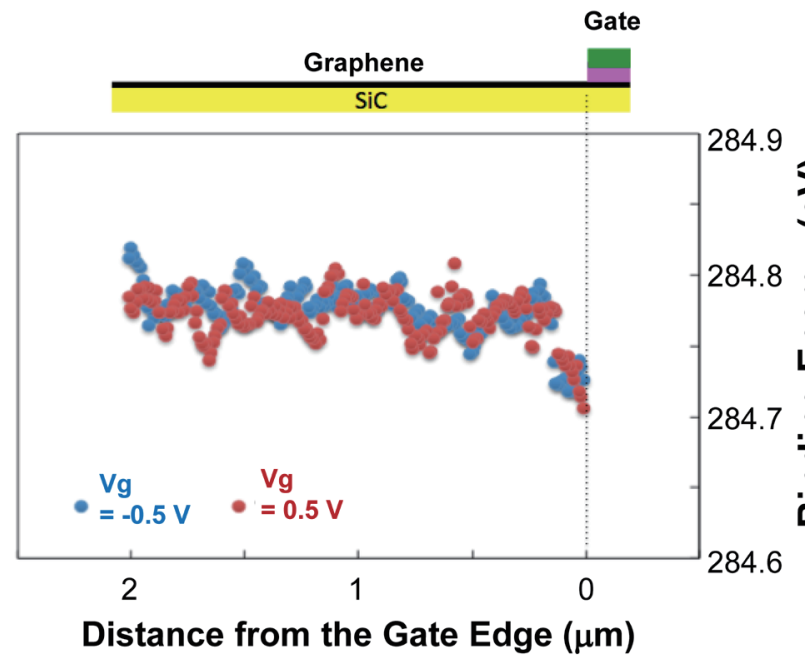

(a)

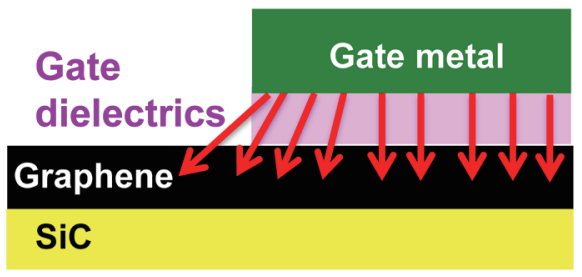

(b)
Not measured Measured

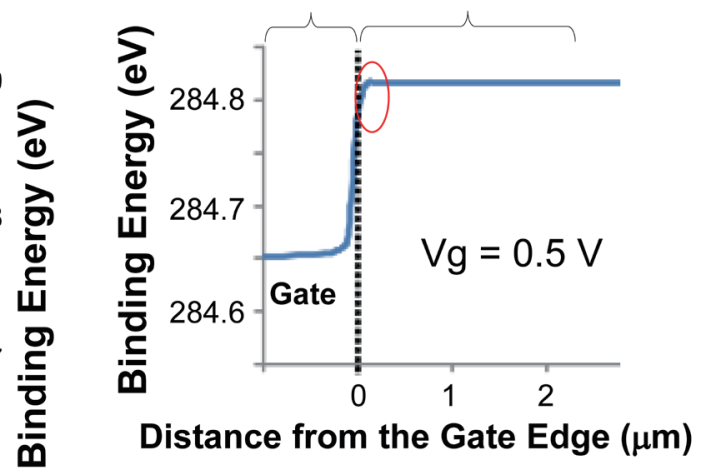

\section{Distance from the Gate Edge $(\mu \mathrm{m})$}

(c)

Fig. 3. (Color online) (a) Spatial change in the binding energy of the graphene peak near the source electrode, (b) schematics of doping by the stray gate field, and (c) calculations of the spatial changes in binding energies at the gate biases of 0.5 and $-0.5 \mathrm{~V}$.

The decrease in electron density is considered to be due to the permeated electric field. The main role of the applied gate bias is to vary the carrier concentration in graphene lying just beneath the gate dielectrics by producing an electric field normal to the surface. However, in fact, the gate electric field is permeated slightly. Part of the electric line of force near the gate edge extends to graphene outside the gate electrode, as schematically shown in Fig. 3(b). This permeated electric field changes the density of doped electrons in graphene just outside the gate edge. For a quantitative examination of our idea, we roughly calculated a spatial variation in the binding energy of graphene from the gate to source electrodes, as shown in Fig. 3(c). In the calculation, we assumed electron doping of $5 \times 10^{12} \mathrm{~cm}^{-2}$ in graphene before applying the gate bias.

In this calculation, $0.96 \mu \mathrm{F} / \mathrm{cm}^{2}$ was used for the gate oxide $\left(\mathrm{Al}_{2} \mathrm{O}_{3}\right)$ capacitance $\left(C_{o x}\right)$. $C_{o x}$ was estimated by $5 \mathrm{~nm}$ for the thickness of $\mathrm{Al}_{2} \mathrm{O}_{3}: 5 \mathrm{~nm}$ and 5.4 for the relative dielectric constant. The simulated binding energy away from the gate electrode, i.e., the region not subject to the gate bias, is consistent with the experimental data. The potential distribution of graphene under the applied gate bias was calculated by using the device simulator Atlas, which has been used for determining device characteristics. ${ }^{(6)}$ Then, the spatial distribution of the 
binding energy was calculated by using the calculated potential that determines $E_{F}$ [Fig. 3(c)]. As a result, the calculation reproduces the observed change in the binding energy of graphene just near the gate edge. Furthermore, the calculation supports the experimental fact that the change in binding energy is independent of the gate bias. Thus, we conclude that the stray gate electric field induces the doping just near the gate edge.

\section{Conclusions}

Our operando photoelectron nanospectroscopy demonstrated that unintentional doping due to the contact with metal and the stray gate electric field occurs in EG-GFET. This finding will serve as the key for high-performance EG-GFET. We believe that operando $\mathrm{X}$-ray spectromicroscopy is surely useful for clarifying the operation mechanisms of advanced electronic and MEMS devices.

\section{Acknowledgments}

The fabrication of EG-GFET was carried out in the cleanrooms of nanospin facilities of Research Institute of Electrical Communications with the help of Professors Shigeo Sato and Masao Sakuraba. Part of this work was supported by KAKENHI (18K19011 and 16H06361), SCOPE of the Ministry of Internal Affairs and Communications, SEI Group CSR Foundation, and RIEC cooperation projects. The operando photoelectron nanospectroscopy was carried out at BL07LSU of SPring-8 (2014B7472 and 2017B7545).

\section{References}

1 K. S. Novoselov, A. K. Geim, S. V. Morozov, D. Jiang, Y. Zhang, S. V. Dubonos, I. V. Grigorieva, and A. A. Firsov: Science 306 (2004) 666. https://doi.org/10.1126/science.1102896

2 Y. Zhang, Y.-W. Tan, H. L. Stormer, and P. Kim: Nature 438 (2005) 201. https://doi.org/10.1038/NPHYS2104

3 F. Schwierz: Nat. Nanotechnol. 5 (2010) 487. https://doi.org/10.1038/nnano.2010.89

4 M.-H. Jung, G.-H. Park, T. Yoshida, H. Fukidome, T. Suemitsu, T. Otsuji, and M. Suemitsu: Proc. IEEE 101 (2013) 1603. https://doi.org/10.1109/JPROC.2013.2258651

5 H. Fukidome, K. Nagashio, N. Nagamura, K. Tashima, K. Funakubo, K. Horiba, M. Suemitsu, A. Toriumi, and M. Oshima: Appl. Phys. Exp. 7 (2014) 065101. https://doi.org/10.7567/APEX.7.065101

6 K. Omika, Y. Tateno, T. Kouchi, T. Komatani, S. Yaegassi, K. Yui, K. Nakata, N. Nagamura, M. Kotsugi, K. Horiba, M. Oshima, M. Suemitsu, and H. Fukidome: Sci. Rep. 8 (2018) 13268. https://doi.org/10.1038/s41598018-31485-4

7 H. Fukidome, M. Kotsugi, K. Nagashio, R. Sato, T. Ohkouchi, T. Itoh, A. Toriumi, M. Suemitsu, and T. Kinoshita: Sci. Rep. 4 (2014) 3713. https://doi.org/10.1038/srep03713

8 G. Kamata, G. Venugoapl, M. Kotsugi, T. Ohkochi, M. Suemitsu, and H. Fukidome: Phys. Status Solidi A (2018) 1800539. https://doi.org/10.1002/pssa.201800539

9 K. V. Emtsev, A. Bostwick, K. Horn, J. Jobst, G. L. Kellogg, L. Ley, J. L. McChesney, T. Ohta, S. A. Reshanov, J. Röhrl, E. Rotenberg, A. K. Schmid, D. Waldmann, H. B. Weber, and Th. Seyller: Nat. Mater. 8 (2009) 203. https://doi.org/:10.1038/NMAT2382

10 H. Fukidome, Y. Kawai, F. Fromm, M. Kotsugi, H. Handa, T. Ide, T. Ohkouchi, H. Miyashita, Y. Enta, T. Kinoshita, Th. Seyller, and M. Suemitsu: Appl. Phys. Lett. 101 (2012) 041605. https://doi.org/10.1063/1.4740271

11 M. Hasegawa, K. Tashima, M. Kotsugi, T. Ohkochi, M. Suemitsu, and H. Fukidome: Appl. Phys. Lett. 109 (2016) 111604. https://doi.org/10.1063/1.4962840 
12 K. Horiba, Y. Nakamura, N. Nagamura, S. Toyoda, H. Kumigashira, M. Oshima, K. Amemiya, Y. Senba, and H. Ohashi: Rev. Sci. Instrum. 82 (2011) 113701. https://doi.org/10.1063/1.3657156

13 H. Fukidome, T. Ide, Y. Kawai, T. Shinohara, N. Nagamura, K. Horiba, M. Kotsugi, T. Ohkochi, T. Kinoshita, H. Kumigashira, M. Oshima, and M. Suemitsu: Sci. Rep. 4 (2014) 5173. https://doi.org/10.1038/srep05173

14 R. Suto, G. Venugopal, M. Suemitsu, K. Tashima, N. Nagamura, M. Oshima, and H. Fukidome: Mater. Res. Express 3 (2016) 075004. https://doi:10.1088/2053-1591/3/7/075004

15 N. Nagamura, K. Horiba, S. Toyoda, S. Kurosumi,T. Shinohara, M. Oshima, H. Fukidome, M. Suemitsu, K. Nagashio, and A. Toriumi: Appl. Phys. Lett 102 (2013) 241604. https://doi.org/10.1063/1.4808083

16 K. V. Emstev, F. Speck, Th. Seyller, and L. Ley: Phys. Rev. B 77 (2008) 155303. https://doi.org/10.1103/ PhysRevB.77.155303

17 K. C. Prince, I. Ulrych, M. Peloi, and B. Ressel, V. Cháb, C. Crotti, and C. Comicioli: Phys. Rev. B 62 (2001) 6866. https://doi.org/10.1103/PhysRevB.62.6866

18 J. Ristein, S. Mammadov, and Th. Seyller: Phys. Rev. Lett. 108 (2012) 246104. https://doi.org/10.1103/ PhysRevLett.108.246104

19 K. Nagashio and A. Toriumi: Jpn. J. Appl. Phys. 50 (2011) 070108. https://doi.org/10.1143/JJAP.50.070108

20 P. A. Khomyakov, G. Giovannetti, P. C. Rusu, G. Brocks, J. van den Brink, and P. J. Kelly: Phys. Rev. B 79 (2009) 195425. https://doi.org/10.1103/PhysRevB.79.195425

21 T. Ando, A. B. Fowler, and F. Stern: Rev. Mod. Phys. 54 (1982) 437. https://doi.org/10.1103/RevModPhys.54.43

\section{About the Authors}

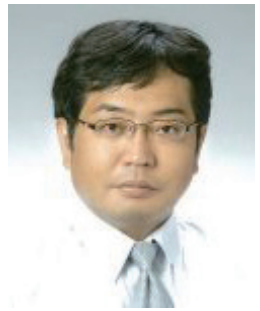

Hirokazu Fukidome received his B.S., M.S., and Ph.D. degrees from Osaka University, Japan, in 1995, 1997, and 2000, respectively. From 2000 to 2001, he was a postdoctoral fellow at AT\&T Bell Laboratories, USA. From 2001 to 2002, he was a postdoctoral fellow at RIKEN, Japan. From 2002 to 2007, he was an assistant professor at Toyota Technological Institute, Japan. From 2008 to 2011, he was an assistant professor at Tohoku University, Japan. Since 2012, he has been an associate professor at Tohoku University, Japan. His research interests are in surface science, crystal growth, MEMS, electrochemistry, X-ray spectroscopy, and high-speed devices.

(fukidome@riec.tohoku.ac.jp)

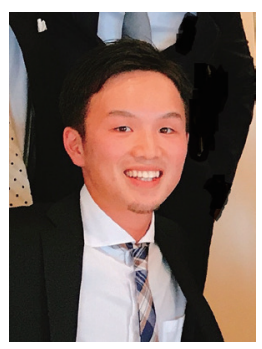

Kazutoshi Funakubo received his B.S. and M.S. degrees from Tohoku University, Japan, in 2012 and 2014, respectively. Since 2014, he has been a project engineer for biomass power plants at Sumitomo Heavy Industries, Ltd. (kazutoshi.funakubo@shi-g.com)

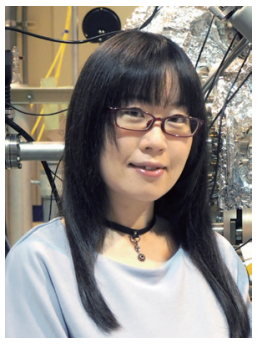

Naoka Nagamura received her B.S., M.S., and Ph.D. degrees from the University of Tokyo, Japan, in 2005, 2007, and 2011, respectively. From 2011 to 2013, she was a postdoctoral fellow at the University of Tokyo, Japan. From 2013 to 2015, she was an assistant professor at Tohoku University, Japan. Since 2015, she has been a researcher at the National Institute for Materials Science, Japan. Since 2017, she has been a JST PRESTO researcher in Japan. Her research interests are the surface/interface physics and synchrotron X-ray spectroscopy analysis. (NAGAMURA.Naoka@nims.go.jp) 


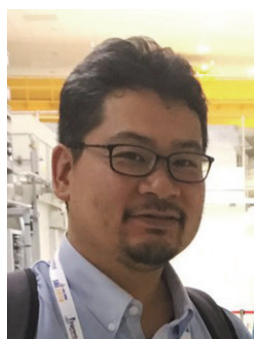

Koji Horiba received his B.S., M.S., and Ph.D. degrees from the University of Tokyo, Japan, in 1998, 2000, and 2003, respectively. From 2003 to 2006, he was a postdoctoral fellow at RIKEN, Japan. From 2006 to 2012, he was an assistant professor and lecturer at the University of Tokyo, Japan. Since 2012, he has been an associate professor at IMSS-KEK, Japan. His research interests are in synchrotron radiation science and photoelectron spectroscopy. (horiba@post.kek.jp)

Yasunori Tateno received his B.S. degree from Kyoto University. He currently engages in research and development of communication devices in Sumitomo Electric Industries.

Masaharu Oshima received his B.S., M.S., and Ph. D degrees from the University of Tokyo, Japan, in 1972, 1974, and 1984, respectively. From 1995 to 2013, he was a full professor at the University of Tokyo, Japan. Since 2013, he has been a project professor and a project researcher at ISSP, University of Tokyo. His research interests are in surface chemistry and synchrotron radiation science. (oshima@sr.t.u-tokyo.ac.jp)

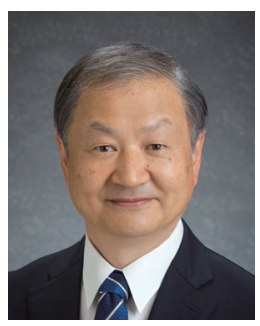

Maki Suemitsu received his B.Eng., M.Eng., and Ph.D. degrees in electronic engineering from Tohoku University, Sendai, Japan, in 1975, 1977, and 1980, respectively. He worked as a research associate from 1980 to 1989, and then as an associate professor from 1990 to 2002 at the Research Institute of Electrical Communication (RIEC), Tohoku University. In 2003, he joined the Center for Interdisciplinary Research, Tohoku University, as a professor, and has been a professor at RIEC since 2008. His research interests include the epitaxy of group-IV materials on Si substrates and their use in devices, which are currently converging in graphene-on-silicon technology. He is the author of more than 200 papers. Prof. Suemitsu is the recipient of the 30th Kumagai Award of the Vacuum Society of Japan. He is a member of the Japan Society of Applied Physics, the Vacuum Society of Japan, the Electrochemical Society, and the Materials Research Society. (suemitsu@riec.tohoku.ac.jp) 